\title{
Sex differences in shock thresholds in rats and gerbils and the day-night cycle
}

\author{
WILLIAM W. BEATTY and GERALD A. HOLZER \\ Department of Psychology, North Dakota State University, Fargo, North Dakota 58102
}

\begin{abstract}
Sex differences in shock thresholds in relation to the day-night cycle were examined in rats and gerbils using a modification of the flinch-jump technique. In rats, males had higher thresholds than females in both day and night tests, but the magnitude of the difference was greater during the night tests. This effect arose because females tested at night had lower thresholds than females tested in the day. Males were little affected by the day-night cycle. In gerbils there was no sex difference in shock thresholds either in day or night tests, but shock thresholds were lower at night than during the day.
\end{abstract}

Studies of behavioral variation in relation to the daynight cycle have typically focused on a very limited sample of behavior. Diurnal differences in locomotor activity and feeding and drinking have been extensively studied (e.g., Nance \& Gorski, 1975; Roper, 1976; Zucker, Rusak, \& King, 1976), but the possibility of cyclic variation in learning and performance with the day-night cycle has received comparatively little attention. The present study examined sensitivity to electric shock in relation to the day-night cycle in rats and gerbils of both sexes.

\section{METHOD}

\section{Animals}

The subjects were 17 male and 17 female albino rats obtained from the Holtzman Company, Madison, Wisconsin, and 17 male and 16 female Mongolian gerbils (Meriones ungiculatus, MON-TUM) obtained from Tumblebrook Farm, West Brookfield, Massachusetts. All of the animals had received previous tests on DRL and shuttlebox avoidance tasks as described elsewhere (Beatty, 1977). At the time of the present tests, the animals were about 7 months old. They were caged singly with free access to food and water in an air-conditioned animal room that was illuminated from $0800-2000 \mathrm{~h}$.

\section{Procedure}

Since all of the animals had previously been trained in active avoidance either in the day or at night, animals were assigned to groups $(\mathrm{N}=8$ or 9 each) that received shock threshold tests during the day $(0900-1100 \mathrm{~h})$ or at night $(2100-2300 \mathrm{~h})$, so that the time of the shock threshold testing and the time of the earlier avoidance tests were not confounded. Shock thresholds were determined by exposing the animals to a single ascending series of $.5-\mathrm{sec}$ scrambled ac shocks on each of 4 test days. The shocks were delivered from a Grason Stadler E700 source to the grid floor (made of bronze welding rod) of a small chamber with walls and a roof made of transparent plastic. For rats the chamber measured $23.5 \times 25.0 \times 23.0 \mathrm{~cm}$ high; for gerbils the chamber measured $15.2 \times 15.3 \times 15.0 \mathrm{~cm}$ high. White noise masked the sound of the shocker.

An observer located in an adjacent room operated the shock source and classified the subject's behavior into one of four mutually exclusive categories: no response, flinch (any detectable

This research was supported by Grant HD 08206 from PHS. movement), shuffle (flinch followed by movement of the feet usually at shock offset), or jump (all four feet leave the grid). On each session testing began at the lowest current the source could deliver $(.05 \mathrm{~mA})$ and was continued by presenting progressively more intense shocks until the animal jumped on two consecutive presentations. Shocks were presented as soon as the animal assumed a posture with all four feet on the grid (normally within 5-20 sec of the preceding trial). Thresholds were computed separately for each response on each day. The threshold was defined as the current required to elicit a particular response reliably (i.e., on two consecutive test trials) and was computed as described elsewhere (Beatty \& Fessler, 1977). Since there were no consistent changes in thresholds across days, average thresholds for flinch, shuffle, and jump were determined for each animal.

\section{RESULTS AND DISCUSSION}

Table 1 describes the mean values for each of the shock thresholds. Overall, gerbils had lower flinch and shuffle thresholds than rats $[F(1,59)=14.91$ and 11.12 , respectively, both ps $<.01]$, but there was no reliable difference on the jump threshold measure $(F=1.11)$. Of greater interest was the pattern of sex differences in relation to the day-night cycle in the two species. As expected from previous work (e.g., Beatty \& Beatty, 1970; Beatty \& Fessler, 1976), female rats had lower shock thresholds than males on all measures. These sex differences in sensitivity to shock occurred during both day and night tests $[\mathrm{F}(1,30)=26.57$, 34.36 , and 40.13 for flinch, shuffle, and jump thresholds, respectively, all ps $<.001]$. However, the differences between male and female rats were greater at night than

Table 1

Mean Shock Thresholds (mA)

\begin{tabular}{lcccccccc}
\hline & \multicolumn{3}{c}{ Rats } & & \multicolumn{3}{c}{ Gerbils } \\
\cline { 2 - 4 } \cline { 6 - 8 } \cline { 6 - 8 } & Flinch & Shuffle & Jump & & Flinch & Shuffle & Jump \\
\hline Males-Day & .155 & .253 & .443 & & .131 & .207 & .397 \\
Males-Night & .152 & .260 & .418 & & .098 & .160 & .274 \\
Females-Day & .125 & .190 & .320 & & .117 & .191 & .370 \\
Females-Night & .097 & .146 & .242 & & .102 & .168 & .301 \\
\hline
\end{tabular}


in the day and this effect arose because in females thresholds at night were generally lower than during the day. Significant differences between groups of females tested at night or during the day were observed on the shuffle and jump threshold measures $[\mathrm{t}(15)=2.77$ and 2.44 , respectively, both ps $<.05]$. On the flinch threshold, the difference between female groups approached significance $[\mathrm{t}(15)=2.01, \mathrm{p}<.10]$. By contrast, shock thresholds of male rats were little affected by the time of testing.

The pattern observed in gerbils was quite different from that seen in rats. There was no sex difference on any of the measures of sensitivity to shock, but in both sexes thresholds were lower at night than in daytime tests for all measures $[F(1,29)=14.05,8.51$, and 9.91 for flinch, shuffle, and jump thresholds, respectively, all ps $<.01]$.

The present finding that shock thresholds of female rats are influenced by the day-night cycle, while those of males are not affected by this variable provides a plausible explanation for the surprising finding that acquisition of shuttlebox avoidance behavior by female rats is much better during the day than at night, while males acquire two-way avoidance responses equally poorly at both stages of the day-night cycle (Beatty, 1977). Previous work in our laboratory (Beatty \& Beatty, 1970) has demonstrated that acquisition of twoway avoidance behavior is impaired in both males and females if the US intensity is increased from 1.0 to $1.5 \mathrm{~mA}$. Since the US intensity of $1.0 \mathrm{~mA}$ used in the experiment examining shuttlebox avoidance in relation to the day-night cycle was near the top of the range of US values that produce optimal acquisition, it seems reasonable, based on the variation of shock thresholds with the day-night cycle in female rats, to suppose that the $1.0-\mathrm{mA}$ US was perceptually more intense at night than in the day for these animals. If this interpretation is correct, then a decrement in shuttlebox avoidance by female rats tested at night is understandable if a moderately intense US is used. Since male rats do not exhibit diurnal variations in shock thresholds, no influence of the day-night cycle on their avoidance behavior would be expected and none was observed
(Beatty, 1977). However, recent unpublished data from our laboratory demonstrate that CS conditions interact with the sex of the subject, the US intensity and the stage of the day-night cycle to determine shuttlebox avoidance performance. Thus, increased sensitivity to shock during the night may influence avoidance performance adversely, but only under certain testing conditions.

The absence of sex differences in pain sensitivity in gerbils parallels our earlier finding that hamsters do not exhibit the same sexual dimorphism on this behavior as has been consistently observed in rats (Beatty \& Fessler, 1977). Again the desirability of a comparative examination of sex differences in behavior is indicated.

\section{REFERENCES}

BeATtY, W. W. Sex differences in DRL and active avoidance behaviors in the rat depend upon the day-night cycle. Bulletin of the Psychonomic Society, 1977, 10, 95-97.

BeAtTy, W. W., \& Beatty, P. A. Hormonal determinants of sex differences in avoidance behavior and reactivity to electric shock in the rat. Journal of Comparative and Physiological Psychology, 1970, 73, 446-455.

BeAtTy, W. W., \& Fessler, R. G. Ontogeny of sex differences in open field behavior and sensitivity to electric shock. Physiology and Behavior, 1976, 16, 413-417.

BeatTy, W. W., \& Fessler, R. G. Sex differences in sensitivity to electric shock in rats and hamsters. Bulletin of the Psychonomic Society, 1977, 10, 189-190.

NANCE, D. M., \& Gorski, R. A. Neurohormonal determinants of sex differences in the hypothalamic regulation of feeding behavior and body weight in the rat. Central Control of Eating and Obesity, Pharmacology Biochemistry and Behavior, 1975, 3, Supplement 1, 155-162.

Roper, T. J. Sex differences in circadian wheel running rhythms in the Mongolian gerbil. Physiology and Behavior, 1976, 17, 549-552.

ZuCKer, I., RUSAK, R., \& KING, R. G. Neural bases for circadian rhythms in rodent behavior. In A. H. Riesen \& R. F. Thompson (Eds.), Advances in Psychobiology (Vol. 3). New York: Wiley, 1976.

(Received for publication September 26, 1977.) 